\title{
Blood transfusion related to procedure in adult patients undergoing cardiac surgery
}

\author{
K Nadar, ${ }^{1}$ (D) CS Alphonsus, ${ }^{2}$ (D) A Hardy ${ }^{3}$
}

${ }^{1}$ Department of Anaesthetics, Inkosi Albert Luthuli Central Hospital, Nelson R Mandela School of Medicine, University of KwaZulu-Natal, South Africa

${ }^{2}$ Department of Anaesthesia and Perioperative Medicine, Groote Schuur Hospital, South Africa

${ }^{3}$ Department of Statistical Sciences, University of Cape Town, South Africa

Corresponding author, email: kresen.nadar@gmail.com

Introduction: Blood transfusions are associated with increased adverse outcomes and healthcare costs. While the aim of transfusion is to improve patient outcomes by enhancing tissue oxygenation, this must be weighed against the risks of transfusion-associated complications. Patient blood management programmes (PBM) have been implemented in many developed countries and have shown decreased blood utilisation and improved outcomes. Cardiac surgery is a significant consumer of blood supplies, despite evidence demonstrating the safety of restrictive transfusion strategies. This study aimed to determine the risk of blood transfusion, according to type of surgical procedure, in cardiac surgical patients in a quaternary level referral hospital in South Africa.

Methods: This single centre, prospective observational study conducted over 18 weeks at Inkosi Albert Luthuli Central Hospital investigated the risk of packed red blood cell (PRBC) transfusion from the intraoperative period until discharge in adult patients undergoing elective CABG $(n=30)$, valve surgery $(n=53)$ or redo surgery $(n=11)$ under cardiopulmonary bypass. Poisson regression assessed the effect of type of procedure, age, gender, body mass index, preoperative haemoglobin and EuroSCORE II as risk factors for PRBC transfusion.

Results: Ninety-four patients were recruited. There was no statistically significant association between risk of PRBC transfusion in valve surgery (IRR $0.71 ; 95 \% \mathrm{Cl} 0.51-1 ; p=0.052$ ) and redo surgery (IRR $0.87 ; \mathrm{Cl} 0.49-1.52 ; p=0.621$ ) when compared to CABG. Increased risk of PRBC transfusion was associated with female gender (IRR 1.72;95\% CI 1.24-2.41; $p=0.001$ ) and EuroSCORE II (IRR $1.06 ; 95 \% \mathrm{Cl} 1.02-1.1 ; p=0.001)$. Increasing preoperative haemoglobin levels were associated with a lower risk of PRBC transfusion (IRR 0.89; 95\% Cl 0.82-0.98; $p=0.013$ ). Median number of PRBC units transfused was: 2 units (entire cohort); 3 units (CABG); 2 units (valve surgery); and 3 units (redo surgery).

Conclusions: PRBC transfusion occurred in $80 \%$ of patients undergoing cardiac surgery, however the risk of blood transfusion was not associated with the type of surgical procedure. Implementation of a PBM protocol may decrease the incidence of preoperative anaemia, which was present in half the study population.

Keywords: cardiac anaesthesia, cardiac surgery, patient blood management, blood transfusion, transfusion risk

\section{Introduction}

Cardiac surgery is associated with increased rates and volumes of perioperative blood transfusions, with up to $15 \%$ of stored blood in the USA ${ }^{1}$ and $6 \%$ of stored blood in the UK² utilised during cardiac surgical procedures. Within this population, it has been found that up to $80 \%$ of packed red blood cell (PRBC) transfusions occur in a minority of patients. ${ }^{3}$ The aim of PRBC transfusion is to improve oxygen delivery to tissues to avoid complications associated with tissue hypoxia, however this must be weighed against the risks of blood transfusion. PRBC transfusion in cardiac surgery is associated with an increase in early and late mortality, hospital length of stay, multiorgan failure, infection, thrombosis and cost. ${ }^{4}$ The optimal haemoglobin threshold below which PRBC transfusion is indicated is still unclear. Studies exploring the effects of liberal and restrictive transfusion strategies have yielded conflicting results. Early studies such as the Transfusion Indication Threshold Reduction (TITRe2) trial found an increased mortality associated with a restrictive threshold $\mathrm{Hb}$ $<7.5{\left.\mathrm{~g} . \mathrm{dl}^{-1}\right)}^{-1}$ compared to a liberal threshold $\left(\mathrm{Hb}<9 \mathrm{~g} \cdot \mathrm{dl}^{-1}\right),{ }^{5}$ while the Transfusion Requirements After Cardiac Surgery (TRACS) trial found no difference in 30-day mortality or morbidity when comparing restrictive and liberal transfusion thresholds. ${ }^{6}$ More recently, the Transfusion Requirements in Cardiac Surgery (TRICS III) study showed that a restrictive transfusion strategy is safe in high-risk patients. ${ }^{7}$ Current guidelines recommend that the decision to transfuse be based on the clinical scenario rather than a fixed haemoglobin threshold. ${ }^{8}$ Clinicians should aim to maintain a balance between oxygen delivery and oxygen extraction, by considering the interaction of haematocrit, temperature, oxygen supply, haemodynamics and tissue perfusion. ${ }^{8}$ Observational studies have found significant variation in intraoperative transfusion rates between different practitioners $^{9}$ and institutions. ${ }^{4,10}$ Preoperative risk factors for transfusion include advanced age, dual antiplatelet therapy (DAPT), poor platelet function, anaemia, small body surface area, female gender, non-elective surgery, non-isolated surgery, noncoronary artery bypass graft (CABG) surgery and redo surgery. ${ }^{8}$ Scoring systems incorporating these factors have been designed to aid preoperative estimation of intra- and postoperative blood requirements, however they have not been externally validated and their usefulness in the South African setting is uncertain. 
The scarcity and cost of blood supplies, and the risks associated with transfusion, have prompted the development of patient blood management (PBM) initiatives in many countries. PBM is 'the timely application of evidence-based medical and surgical concepts designed to maintain haemoglobin concentration, optimise haemostasis and minimise blood loss in an effort to improve patient outcome.'11 The three pillars of PBM, which act in concert in the preoperative, intraoperative and postoperative periods, are: (i) optimisation of red cell mass; (ii) minimisation of blood loss and bleeding; and (iii) harnessing and optimising physiological reserve of anaemia. ${ }^{12}$ Despite the many advantages of PBM and its adoption in some developed countries, it has not yet been adopted as a care bundle in South African hospitals. Additional PBM techniques which can be considered in cardiac surgery are the use of minimally invasive extracorporeal circulation circuits (MiECC), biocompatible coated circuits, ultrafiltration and minimisation of haemodilution, topical haemostatics, routine use of antifibrinolytics and haemostatic monitoring. ${ }^{8}$ Point of care thromboelastography-directed transfusion protocols in cardiac surgery have been shown to decrease the incidence and volume of PRBC and blood product transfusion, reoperation rates, postoperative length of stay and 6-month mortality. ${ }^{13,14}$ The implementation of PBM programmes in cardiac surgery has been shown to decrease transfusions, bleeding, costs and improve outcomes. ${ }^{15}$ The first step towards creating a PBM algorithm is to analyse current transfusion practices and identify patients at increased risk for PRBC transfusion.

The aim of this study is to determine the risk and amount of PRBC transfusion, according to type of procedure, in adult patients undergoing cardiac surgery on cardiopulmonary bypass (CPB) at a quaternary referral level hospital in Durban, South Africa. These results may be used to inform the development of a PBM care bundle for cardiac surgery in South Africa.

\section{Methods}

This is a prospective observational cohort-based study, conducted at Inkosi Albert Luthuli Central Hospital (IALCH), a quaternary level referral hospital in Durban, South Africa. Recruitment commenced following ethics approval from the Biomedical Research Ethics Committee (BE001/18) and site permission from the KwaZulu-Natal Department of Health and IALCH. Informed consent was taken the day prior to surgery from the patient or a legally authorised representative. All patients aged 18 or older at the time of surgery, undergoing elective valve or CABG surgery on $C P B$ were eligible for inclusion in the study. Patients undergoing off-pump surgery, emergency surgery, those who refused blood products or refused to take part in the study were excluded from the study. The recruitment period per protocol was 16 weeks, however it was extended by two weeks to recruit more patients undergoing redo surgery. The final recruitment period was from 21 May 2018 to 21 September 2018. Data collection started at the beginning of surgery and patients were followed up until discharge from hospital.

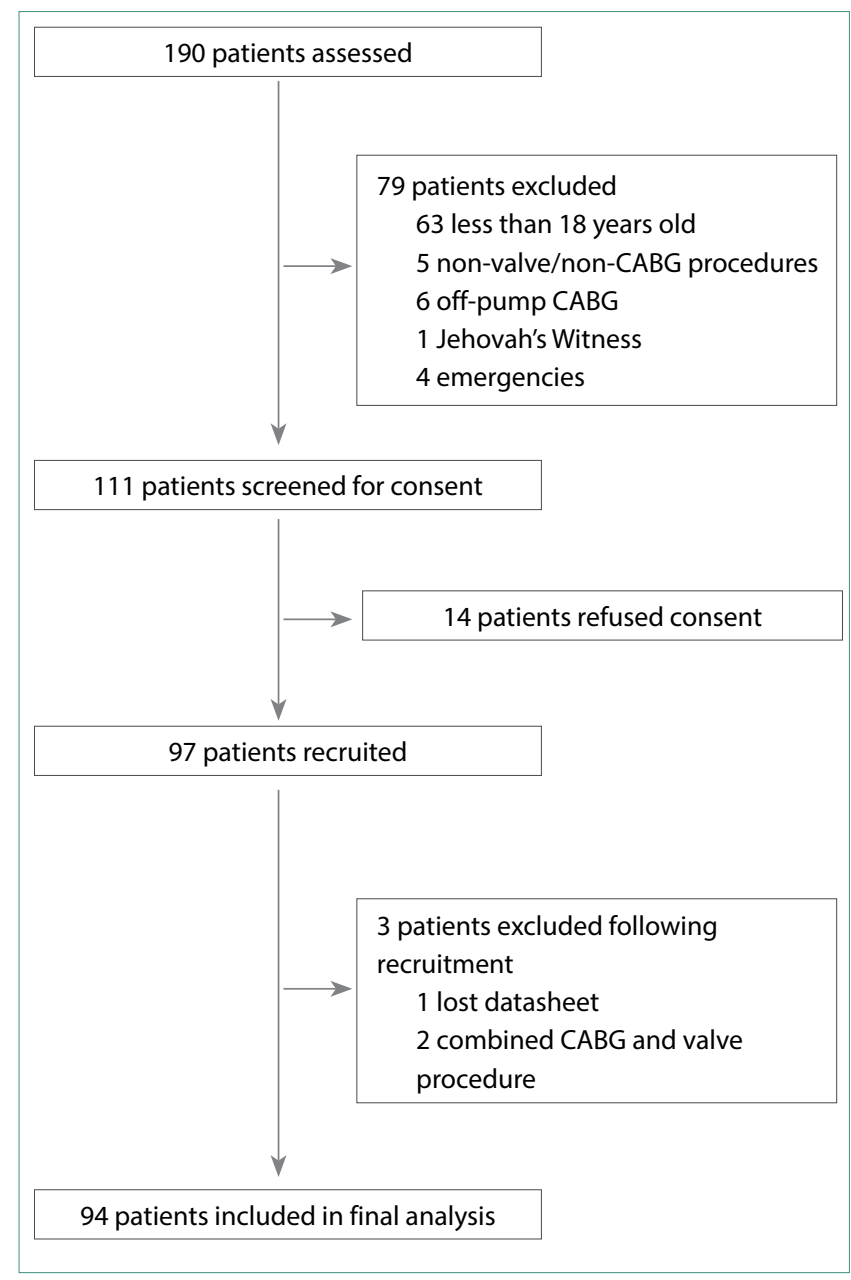

Figure 1: STROBE flow diagram

Figure 1 demonstrates the flow of patients through the recruitment process.

Case report forms (CRFs) were filled in for each patient enrolled in the study. Preoperative demographic data collected included age, gender, body mass index (BMI), EuroSCORE $\|^{16}$ and preoperative haemoglobin concentration. These factors have been shown to correlate with the risk of bleeding and transfusion in cardiac surgery. ${ }^{8,17,18}$ The dependent variable was number of PRBC units transfused to each patient intra- and postoperatively until discharge, and the independent variables were type of procedure with three levels (CABG; valve replacement; and redo surgery), preoperative haemoglobin, BMI, EuroSCORE II, age and gender. Only two patients underwent combined CABG and valve surgery during the recruitment period, so this group was omitted from the study cohort. The cardiac unit at IALCH currently does not utilise a predetermined transfusion trigger, hence the authors did not request for clinicians to document transfusion triggers for patients enrolled in the study, as this may alter transfusion practices during the study period. CRFs were compared with the standard South African National Blood Services (SANBS) transfusion card to ensure that all transfusions were recorded. The study was reported in accordance with the Strengthening the Reporting of Observational Studies in Epidemiology (STROBE) statement. ${ }^{19}$ 
Standard institutional practices at IALCH which may influence bleeding and transfusion are elaborated below. Antiplatelet agents are routinely discontinued one week prior to surgery. Patients awaiting valve surgery at IALCH are anticoagulated with heparin, which is stopped the night before surgery. These practices minimise the influence of preoperative DAPT and anticoagulation on perioperative bleeding. Two units of PRBCs are routinely crossmatched prior to surgery, and availability of blood at the onsite blood bank is confirmed on the morning of surgery. The institution does not have formal indications or haemoglobin triggers for PRBC transfusion, hence the decision to transfuse is based on the judgement of the attending clinician. Tranexamic acid is routinely administered to all patients undergoing CPB, and cell saver processing of shed blood is used in all patients. CPB is initiated following anticoagulation with heparin, and protamine is used at the termination of CPB. Coagulation status is monitored in theatre with point of care activated clotting time (ACT). Viscoelastic point-of-care coagulation testing is not routinely used to monitor and treat coagulation. Moderate hypothermia is practised during $\mathrm{CPB}$, with both nasopharyngeal and bladder temperatures being monitored. Standard volume, non-miniaturised, biocompatible coated circuits are used for CPB. Choice of pump prime solution (crystalloid or blood) is individualised per patient according to surgeon preference.

Statistical analysis was performed with Stata version 16 (College Station, TX, USA: StataCorp, 2019). Incidences of categorical variables were calculated and reported as numbers and percentages. Continuous variables were calculated and presented as medians and interquartile ranges (IQR). The primary outcome was blood usage per type of cardiac surgical procedure. In our analysis of blood usage, the dependent variable was total number of PRBC units transfused, which is a count variable, and the data was skewed to the right, as seen in Figure 2. We used a Poisson regression model with the standard log link function to model the association between mean number of PRBC units transfused and type of surgical procedure while controlling for age, gender, BMI, preoperative haemoglobin and EuroSCORE II. To assess the appropriateness of the link function, the TukeyPregibon link test was performed and the results suggested that the model is well specified. The Pearson dispersion statistic as well as the boundary likelihood ratio test were used to check for overdispersion in the model. The Pearson dispersion statistic was 1.406 for the Poisson model. The likelihood ratio test involves

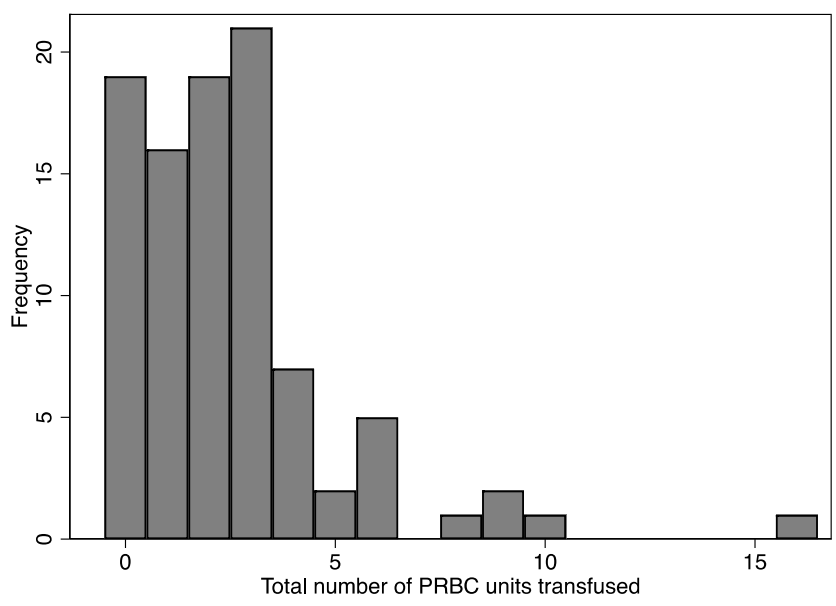

Figure 2: Frequency of PRBC transfusions

evaluating whether the value of the heterogeneity parameter in the negative binomial model is in fact Poisson, that is, with a heterogeneity parameter of 0 . The estimate of the heterogeneity parameter, alpha, was not large enough to warrant use of the negative binomial model ( $\alpha=0.07$, standard error $=0.068$, chibar2(01) $=1.45, p=0.114)$. Predictors were evaluated for multicollinearity using variance inflation factors (VIF) and all had VIFs less than 5, suggesting no serious violation of multicollinearity. Results from Poisson regression are presented as incidence rate ratios (IRR) with $95 \%$ confidence intervals. For all analyses, $p<0.05$ was considered statistically significant.

\section{Results}

One hundred and ninety cardiac surgical procedures were performed at IALCH during the recruitment period. Following application of the exclusion criteria, 97 patients were recruited (Figure 1). Two patients underwent combined CABG and valve procedures, which was too few to include in the final analysis, and one CRF was lost. Ninety-four patients formed the final cohort, of which 30 (31.91\%) underwent CABG, 53 (56.38\%) had valve surgery, and 11 (11.7\%) had redo procedures (all of which were redo valve surgery). The baseline characteristics of the study cohort are summarised in Table I. The median age and preoperative haemoglobin of the CABG subgroup is higher than the valve and redo subgroups and the entire cohort; while the median EuroSCORE II, an indicator of perioperative mortality in cardiac surgery, is highest in the redo subgroup.

Table I: Patient characteristics

\begin{tabular}{|c|c|c|c|c|}
\hline Factor & Total & CABG* & Valve & Redo \\
\hline $\mathrm{n}+$ & 94 & $30(31.91 \%)$ & $53(56.38 \%)$ & $11(11.7 \%)$ \\
\hline Sex: female count (\%) & $50(53.9 \%)$ & $8(26.67 \%)$ & $34(64.15 \%)$ & $8(72.73 \%)$ \\
\hline Age (years) & $47(30-51)$ & $58(53-63)$ & $35(27-53)$ & $28(21-44)$ \\
\hline $\mathrm{BMI} \neq\left(\mathrm{kg} \cdot \mathrm{m}^{-2}\right)$ & $24.68(21.43-29.98)$ & $26.04(23.5-29.68)$ & $23.64(20.9-30.2)$ & $23.7(19.3-27.85)$ \\
\hline EuroSCORE II (\%) & $1.6(0.98-2.79)$ & $1.38(0.83-1.89)$ & $1.5(0.98-2.36)$ & $3.71(2.99-4.85)$ \\
\hline Preoperative $\mathrm{Hb} \S$ (g. $\mathrm{dl}^{-1}$ ) & $12.9(11.9-14.4)$ & $13.6(12.4-14.7)$ & $12.7(11.8-13.8)$ & $11.7(10.6-13.8)$ \\
\hline
\end{tabular}

*CABG - coronary artery bypass graft; $\uparrow \mathrm{n}$ - number; $¥ \mathrm{BMI}$ - body mass index; $\S \mathrm{Hb}$ - haemoglobin Age, BMI, EuroSCORE II and preoperative $\mathrm{Hb}$ reported as median and interquartile range 


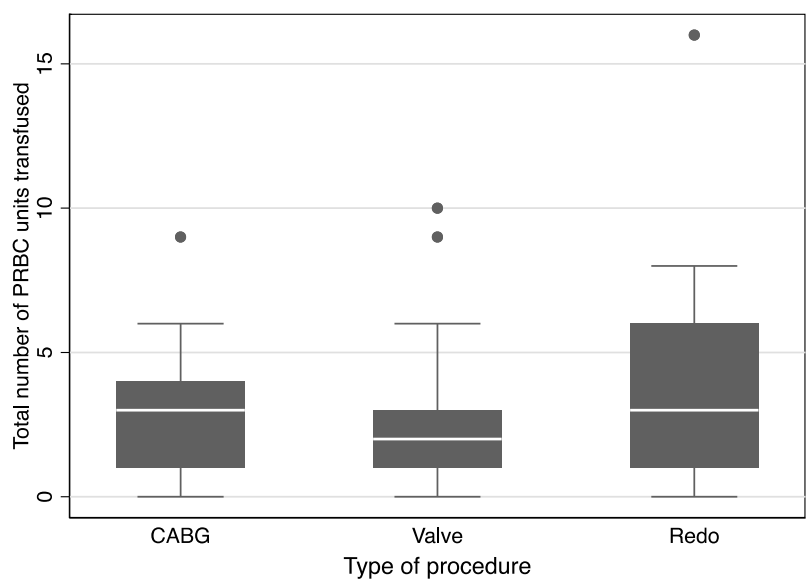

Figure 3: Box and whisker plot of number of PRBC transfusions per type of procedure
Among the patients who were transfused, those undergoing CABG were transfused a larger volume of blood (median 3 units; IQR 1-4) when compared to the patients undergoing valve surgery (median 2 units; IQR 1-3), as shown in Table II and Figure 3. However, the proportion of patients exposed to PRBC transfusion in the CABG subgroup (76.67\%) was lower than that of valve surgery subgroup (79.25\%). Redo surgery had the largest proportion of patients transfused $(90.91 \%)$ and volume of PRBCs transfused per patient (median 3 units; IQR 1-6). Despite these findings, the confounding effects of patient factors on PRBC transfusion must be adjusted for, in order to estimate the association between type of procedure and risk of PRBC transfusion. During the intraoperative phase, PRBC transfusion occurred in $76.6 \%$ ( $n=72$ ) of patients, while in the postoperative phase PRBC transfusion occurred in $24.47 \%(n=23)$ of patients.

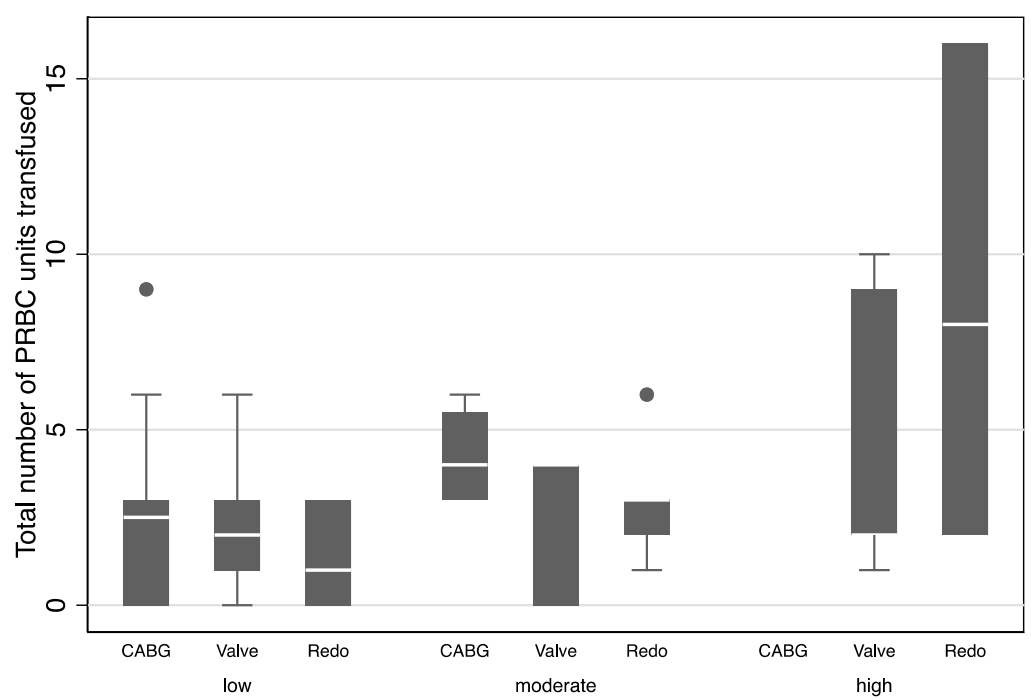

Type of procedure according to EuroSCORE II risk categories

Figure 4: Box and whisker plot of transfusions per type of surgical procedure according to EuroSCORE II risk category Low $<3 \%$; moderate $3-5 \%$; high $>5 \%$

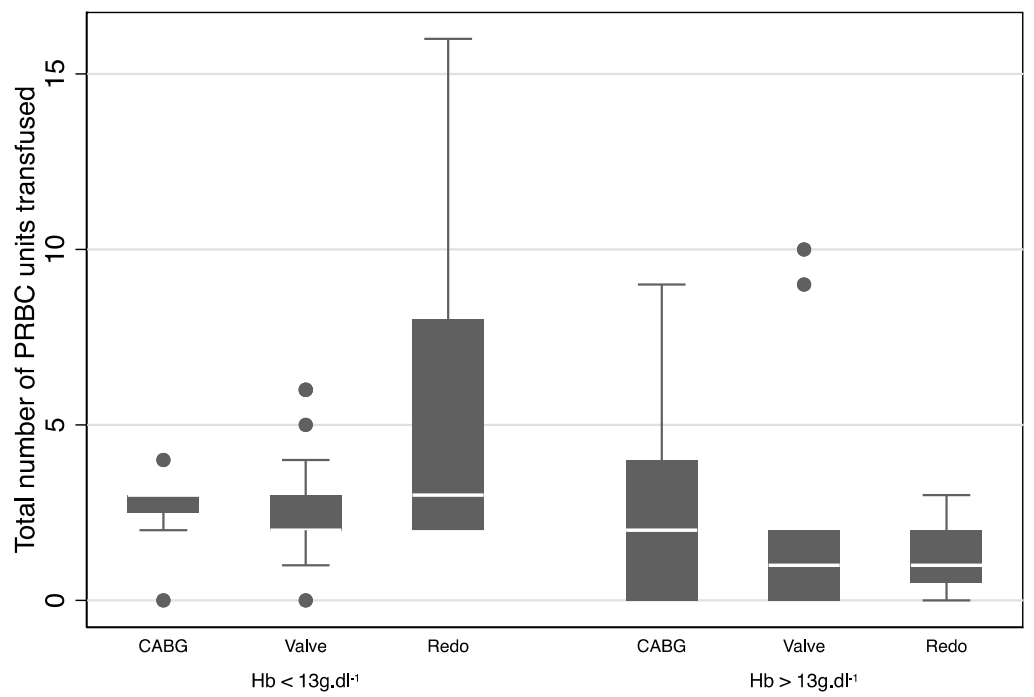

Type of procedure according to preoperative haemoglobin

Figure 5: Box and whisker plot of transfusions per type of surgical procedure according to preoperative haemoglobin levels
PRBC transfusion per type of surgical procedure was also analysed according to subcategories of their BMI, EuroSCORE II, and preoperative haemoglobin (Table III). High-risk EuroSCORE II (Figure 4) and low preoperative haemoglobin subcategories had higher transfusion volumes than the other subcategories (Figure 5). Preoperative anaemia ( $\mathrm{Hb}$ $<13{\mathrm{~g} . \mathrm{dl}^{-1}}^{-1}$ had a prevalence of $53.19 \%(n=50)$ in the study cohort, $40 \%(n=12)$ in CABG patients, $58.49 \%(n=31)$ in valve replacement patients and $63.64 \%(n=7)$ in patients undergoing redo surgery (Table III).

A Poisson regression analysis was done to adjust for the impact of patient factors on PRBC transfusion according to type of surgical procedure while controlling for preoperative haemoglobin, BMI, EuroSCORE II, age and gender. The Poisson model showed decreased IRRs of transfusion in both valve surgery, IRR 0.71 (or $29 \%$ fewer units; $95 \% \mathrm{Cl} 0.51$ $1 ; p=0.052$ ), and redo surgery, IRR 0.87 (or $13 \%$ fewer units; $95 \% \mathrm{Cl} 0.49-1.52 ; p=0.621$ ), which were not statistically significant when compared to CABG. EuroSCORE II, female sex, and age were significantly associated with greater frequency of PRBC transfusion, while preoperative haemoglobin had a statistically significant negative association with PRBC transfusion (Table IV). The model fit was evaluated by considering the observed versus the predicted counts (Figure 6). The model slightly underpredicted number of units transfused for zero, three, six, and nine units transfused and similarly slightly overpredicted number of units transfused for one, four, five and seven units transfused. Predicted number of units greater than 10 fit well with the observed values from the data.

\section{Discussion}

This prospective, observational trial of blood transfusion in adult elective cardiac surgery 
Table II: PRBC transfusions per type of surgical procedure

\begin{tabular}{lccc}
\hline & Patients not transfused & Patients transfused & Units transfused \\
\hline Total & $19(20.21 \%)$ & $75(79.79 \%)$ & $2(1-3)$ \\
CABG & $7(23.33 \%)$ & $23(76.67 \%)$ & $3(1-4)$ \\
Valve & $11(20.75 \%)$ & $42(79.25 \%)$ & $2(1-3)$ \\
Redo & $1(9.09 \%)$ & $10(90.91 \%)$ & $3(1-6)$ \\
\hline
\end{tabular}

PRBC units transfused presented as median and interquartile range

Table III: Number of PRBC units transfused per procedure, according to predictor subgroups

\begin{tabular}{|c|c|c|c|c|c|c|c|}
\hline & & \multicolumn{2}{|r|}{ CABG } & \multicolumn{2}{|c|}{ Valve } & \multicolumn{2}{|r|}{ Redo } \\
\hline & & $n$ & Units transfused & $n$ & $\begin{array}{c}\text { Units } \\
\text { transfused }\end{array}$ & $n$ & $\begin{array}{c}\text { Units } \\
\text { transfused }\end{array}$ \\
\hline \multirow[t]{3}{*}{ BMI } & underweight*/normal† & 11 & $3(3-5)$ & 29 & $1(0-2)$ & 8 & $3(1.5-4.5)$ \\
\hline & overweight‡ & 19 & $2(0-3)$ & 24 & $2.5(0-3)$ & 3 & $2(1-8)$ \\
\hline & low§ & 26 & $2.5(0-3)$ & 43 & $2(1-3)$ & 3 & $1(0-3)$ \\
\hline \multirow[t]{2}{*}{ EuroSCORE II } & moderate $\|$ & 4 & $4(3-5.5)$ & 3 & $4(0-4)$ & 5 & $3(2-3)$ \\
\hline & high** & 0 & $\mathrm{~N} / \mathrm{A}$ & 7 & $2(7)$ & 3 & $8(14)$ \\
\hline \multirow[t]{2}{*}{ Preoperative $\mathrm{Hb}$} & $<13 \mathrm{~g} \cdot \mathrm{dl}^{-1}$ & 12 & $3(2.5-3)$ & 31 & $2(2-3)$ & 7 & $3(2-8)$ \\
\hline & $>13 \mathrm{~g} \cdot \mathrm{dl}^{-1}$ & 18 & $2(0-4)$ & 22 & $1(0-2)$ & 4 & $1(0.5-2)$ \\
\hline
\end{tabular}

PRBC units transfused reported as median and interquartile range; $n$ - number of patients

BMI: *underweight $<18.5 \mathrm{~kg} \cdot \mathrm{m}^{-2}$; tnormal $18.5-24.9 \mathrm{~kg} \cdot \mathrm{m}^{-2} ;$ łoverweight $\geq 25 \mathrm{~kg} \cdot \mathrm{m}^{-2}$

EuroSCORE II: Slow < 3\%; ||moderate 3-5\%; **high > 5\%

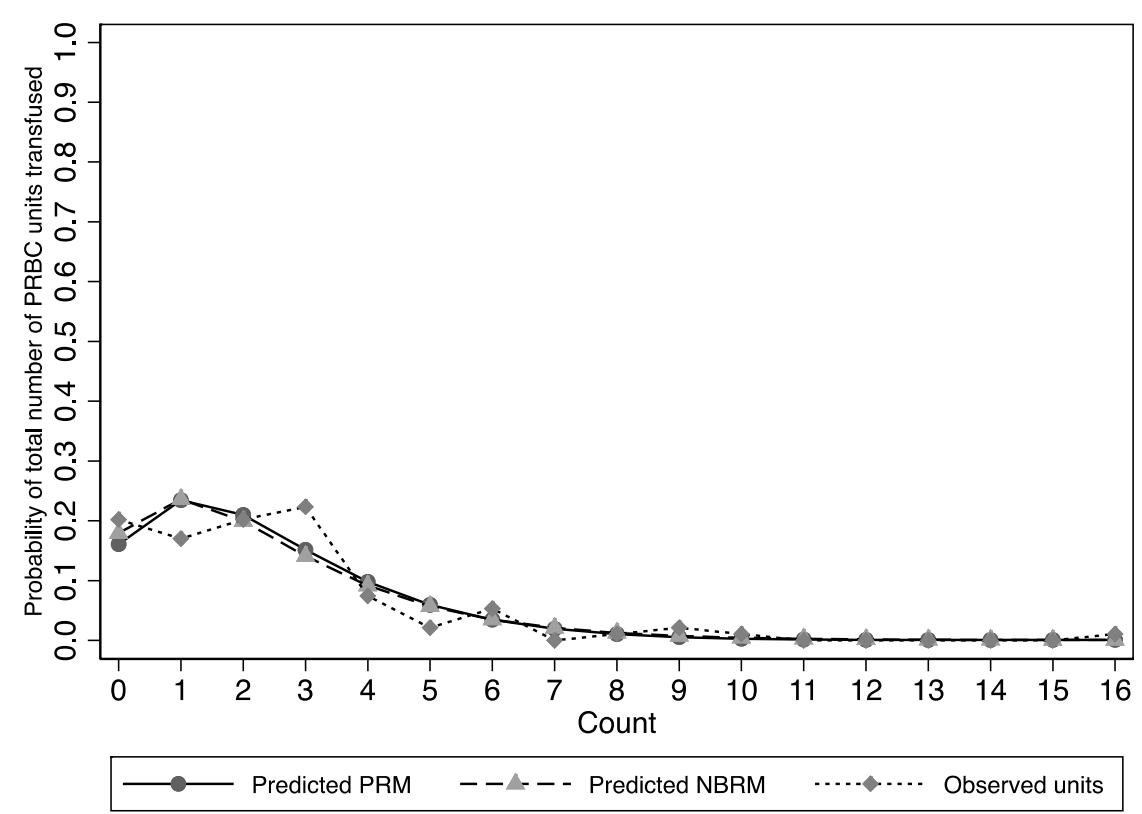

Figure 6: Count models for total number of PRBC units transfused: observed versus predicted probabilities

PRM - Poisson regression model; NBRM - negative binomial regression model

Table IV: Poisson regression analysis of transfusion risk

\begin{tabular}{|c|c|c|c|c|c|}
\hline Total units transfused & Incidence rate ratio & Standard error & $\mathbf{z}$ & $p$ & $\mathbf{9 5} \%$ confidence interval \\
\hline \multicolumn{6}{|l|}{ PROCEDURE } \\
\hline Valve & 0.71 & 0.12 & -1.94 & 0.052 & $0.51-1$ \\
\hline Redo & 0.87 & 0.25 & -0.49 & 0.621 & $0.49-1.52$ \\
\hline \multicolumn{6}{|l|}{ PATIENT FACTORS } \\
\hline EuroSCORE II & 1.06 & 0.02 & 3.26 & 0.001 & $1.02-1.1$ \\
\hline Preoperative haemoglobin & 0.89 & 0.04 & -2.49 & 0.013 & $0.82-0.98$ \\
\hline BMI & 0.98 & 0.01 & -1.84 & 0.066 & $0.95-1$ \\
\hline Female & 1.72 & 0.29 & 3.2 & 0.001 & $1.24-2.41$ \\
\hline Age & 1.02 & 0.01 & 3.56 & $<0.01$ & $1.01-1.03$ \\
\hline
\end{tabular}

Log likelihood - -165.2 
patients on $\mathrm{CPB}$ reports that there is no significant difference in the incidence of the number of PRBC units transfused between CABG, valve surgery (IRR $0.71 ; 95 \% \mathrm{Cl} 0.51-1 ; p=0.052)$ and redo surgery (IRR $0.87 ; 95 \% \mathrm{Cl} 0.49-1.52 ; p=0.621$ ), as per the Poisson regression analysis. This equivalence in transfusion risk between CABG and valve surgery is in keeping with the TRACK ${ }^{20}$ and ACTA-PORT ${ }^{17}$ scoring systems, which do not distinguish between the two types of procedures in their transfusion risk prediction models. In contrast to our findings, the TRUST ${ }^{21}$ score and Goudie et al. ${ }^{18}$ found redo cardiac surgery to carry a higher risk for PRBC transfusion and massive blood transfusion than firsttime cardiac surgery. Surgical challenges commonly associated with redo surgery, such as difficulty accessing the heart, injury to the great vessels and prolonged CPB time are thought to contribute to the increased bleeding and transfusion risk that is usually seen in this group of patients. ${ }^{22}$ During the recruitment period, all patients who had redo surgery underwent redo valve surgery, with no patients undergoing redo CABG. Although not statistically significant, redo surgery had a higher IRR for PRBC transfusion than first time valve surgery in our cohort. While Goudie et al. ${ }^{18}$ and the TRUST ${ }^{21}$ group do not elaborate on patient characteristics of their cohorts that underwent redo cardiac surgery, their populations may have included a wider variety of surgical procedures.

While our initial analysis (Figure 3) showed that patients undergoing $C A B G$ were transfused more than patients undergoing valve surgery, this was no longer demonstrated after adjusting for age, gender, BMI, EuroSCORE II and preoperative haemoglobin. The median age of the CABG group (58 years) was higher than that of both the valve (35 years) and redo (28 years) groups, which may be a contributing factor towards the higher unadjusted transfusion rates in CABG patients. Although our model showed age as a statistically significant determinant of PRBC transfusion, the authors believe that this is not a clinically significant risk factor as its IRR of 1.02 represents only $2 \%$ more PRBC units transfused. Our finding is corroborated by Goudie et al. ${ }^{18}$ who found a 1.29 increased odds of transfusion per 10 year age progression. The higher unadjusted transfusion rate seen in redo surgery (Figure 3), may be due to this group's higher EuroSCORE II and lower preoperative haemoglobin, both of which are predictors of PRBC transfusion. ${ }^{17,18,20,21}$

Wide interinstitutional variation in transfusion practices have been observed, with transfusion rates ranging from $8-93 \% .{ }^{10}$ Our cohort had an $80 \%$ overall incidence of PRBC transfusion, which is comparable to an incidence of $77.3 \%$ observed at another South African hospital. ${ }^{23}$ Potential reasons for our cohort's high incidence of transfusion were not examined in the study and are speculative and probably multifactorial. It may be attributable to variation in clinician practices; the lack of a formal blood transfusion protocol being used in the unit; inadequate use of PBM strategies; or a high-risk patient profile.

In comparison with a Canadian study conducted in $1991^{9}$ which reported mean numbers of PRBCs transfused for CABG (2.8 units), valve surgery (3 units) and redo surgery (3.5-6.3 units), our cohort has seen a decrease in the number of PRBC units transfused per patient. This may be attributable to improved surgical technique and blood conservation strategies.

An increase in blood utilisation was seen with increasing EuroSCORE II risk across all procedures (Figure 4), and the Poisson model revealed EuroSCORE II to have a statistically significant IRR of 1.06 (or $6 \%$ more units) for blood transfusion. One patient with a high EuroSCORE II (22\%) was responsible for the largest number of PRBC units (16 units) transfused in our patient cohort. However, the interplay of other factors, such as preoperative anaemia, low BMI, surgical difficulties and coagulopathy associated with massive blood transfusion, may have contributed to this outlying value. A high blood utilisation was seen in patients with high EuroSCORE II, despite the findings of TRICS III which demonstrated that a restrictive transfusion strategy that targets a haemoglobin concentration of $7.5 \mathrm{~g} \cdot \mathrm{dl}^{-1}$ was noninferior to a liberal strategy, where a haemoglobin concentration less than $9.5 \mathrm{~g} . \mathrm{dl}^{-1}$ prompted transfusion, in moderate to high-risk patients. ${ }^{7}$ This may demonstrate clinician discomfort with accepting lower haemoglobin transfusion thresholds, or a delay in the translation of recent research into clinical practice.

High BMI and body weight is a known protective factor against bleeding and blood transfusion in cardiac surgery. ${ }^{18,20,21,24}$ Production of plasminogen activator inhibitor- 1 by adipocytes is a postulated mechanism for the decreased bleeding seen in obese patients. ${ }^{25}$ With obesity being a component of the metabolic syndrome, it is unsurprising that patients undergoing CABG had the highest median BMI in our patient cohort (26.04kg. $\left.\mathrm{m}^{-2}\right)$. The Poisson regression model did not, however, find $\mathrm{BMI}$ to be an important predictor of transfusion risk in our cohort.

In line with previous studies, our study showed that low preoperative haemoglobin concentration had a statistically significant effect on increased blood utilisation and risk of blood transfusion (Table IV). Preoperative anaemia was present in $53.19 \%$ of patients in our cohort, which is comparable to the $47 \%$ prevalence of anaemia in the non-obstetric, non-cardiac surgical population in South Africa. ${ }^{26}$ As stated before, allogenic blood transfusion carries with it risks of acute kidney injury, acute lung injury, increased graft occlusion, ischaemia, infection, mortality and increased cost. ${ }^{27}$ Preoperative treatment of anaemia may mitigate these risks and decrease the risk of PRBC transfusion. Strategies outlined in PBM programmes, such as IV iron therapy with or without erythropoietin, ${ }^{8}$ may be considered, but these have not been implemented in our unit. Future studies, such as the ITACS (IV iron for Treatment of Anaemia before Cardiac Surgery) Trial, are expected to provide further insight into the role of intravenous iron therapy prior to cardiac surgery.

We observed a wide range in the number of units of blood transfused, from 0 to 16, with the data skewed to the right (Figure 2 ), suggesting that a minority of patients undergo large volume 
blood transfusion. Transfusion risk scoring systems would be beneficial in identifying these patients for the implementation of additional PBM techniques to decrease perioperative bleeding and blood requirements.

The available transfusion risk scoring systems, such as ACTAPORT, ${ }^{17}$ TRACK, ${ }^{20}$ TRUST $^{21}$ and Goudie et al., ${ }^{18}$ are not validated in the South African setting. We postulated that the clinical profile of patients undergoing the different types of cardiac surgical procedures would be different in our population compared to the population used in the development of these scoring systems, and that this may affect their use in our clinical environment. In Europe, the Euro Heart Survey found degenerative disease to account for $63 \%$ of patients with valvular heart disease (VHD), ${ }^{28}$ while in South Africa, the Heart of Soweto study found that degenerative disease accounts for $23 \%$ and rheumatic heart disease accounts for $71 \%$ of VHD. ${ }^{29}$ South African patients with VHD are younger, having a median age of $43,{ }^{29}$ compared to a mean age of $>50$ years in Europe. ${ }^{28}$ Despite this, an equivalence in transfusion risk between CABG and valve surgery was demonstrated in our study, which is in concordance with the transfusion risk scoring systems. This makes it more likely that such transfusion risk scoring tools may be used reliably in the South African environment to plan the procurement of allogenic blood products.

The results of our study suggest that the development and implementation of an institution-specific patient blood management protocol may be beneficial to patient care in our institution. Considering that the incidence of intraoperative PRBC transfusion (76.6\%) far exceeded postoperative PRBC transfusion (24.47\%), PBM strategies targeted at decreasing intraoperative transfusions should be prioritised. Decreased exposure to allogenic blood would decrease the risk of adverse outcomes associated with perioperative anaemia and PRBC transfusion, decrease hospital-associated costs of PRBC transfusion, and conserve blood supplies. Our study analysed current transfusion practices and established a baseline against which the results of future interventions can be compared.

\section{Strengths}

This was a prospective, observational study, and very few datapoints were required to be filled into the CRF, resulting in minimal incomplete CRFs. We had a large case load during the study period. While many studies on transfusion in cardiac surgery have emerged from the developed world, our study has provided a contribution from the South African clinical context.

\section{Limitations}

This is a single centre study, hence the findings are influenced by institution-specific transfusion attitudes and the profile of patients who are served by the hospital. As with observational studies, confounding bias may have influenced the factors investigated, and the Hawthorne effect cannot be excluded. Patients in the redo group are all redo valve surgery - our findings may have differed if redo CABG patients presented during the recruitment period. Events on CPB which may influence coagulation and transfusion, such as duration of $\mathrm{CPB}$, circuit prime, difficulty separating from $C P B$, and technical difficulties during surgery were not taken into account in our analysis. Although temperature is monitored during $\mathrm{CPB}$, we did not investigate the relationship between hypothermia, a known risk factor for bleeding, and transfusion. Intraoperative blood loss and chest tube drainage, which contribute towards increased transfusions, were not recorded. Volumes of crystalloid infusions administered were not documented. The use of other blood products, such as plasma, cryoprecipitate and platelets used to treat coagulopathy or empirically in massive blood transfusion did not factor into our analysis. IALCH does not currently have a protocol to manage bleeding and transfusion in cardiac surgery, hence these decisions are individualised according to patient profile and vary between different clinicians. When PRBC transfusion occurred, the indication, the trigger and the posttransfusion haemoglobin level achieved at the time of discharge were not analysed. Patients may have been unevenly exposed to specific blood conservation strategies, which may act as confounders in our analysis.

\section{Conclusion}

This observational study suggests that there is no difference in the risk of $P R B C$ transfusion between elective $C A B G$, valve surgery and redo cardiac surgery in adult patients on $\mathrm{CPB}$ at IALCH. Other factors, such as increases in EuroSCORE II, low preoperative haemoglobin, female gender and advancing age have been shown to be risk factors for PRBC transfusion in our setting. Our study suggests that, in line with data from centres in South Africa and other countries, cardiac surgery is a major consumer of blood stores in our setting. PBM programmes will aid in preoperatively identifying patients with risk factors for PRBC transfusion. Preoperative risk factor modification and the perioperative implementation of additional blood conservation strategies, that are not part of routine care, should be offered to patients at risk for PRBC transfusion. Preoperative anaemia, a modifiable risk factor for PRBC transfusion, can be addressed by both large-scale public health programmes and in the preoperative optimisation of cardiac surgery patients. In a developing country that has a significant burden of trauma, blood shortages and health budget constraints, the development of PBM programmes to conserve blood stores and avoid the potential adverse outcomes associated with PRBC transfusions, is in the public interest.

\section{Conflict of interest}

The authors declare no conflict of interest.

\section{Funding source}

None.

\section{Ethics approval}

Ethics approval was obtained from the Biomedical Research Ethics Committee (BE001/18). 


\section{ORCID}

\section{K Nadar (iD) https://orcid.org/0000-0002-3753-4300} CS Alphonsus (iD https://orcid.org/0000-0002-0553-0241

\section{References}

1. Zbrozek A, Magee G. Cost of bleeding in trauma and complex cardiac surgery. Clin Ther. 2015;37(9):1966-74. https://doi.org/10.1016/j.clinthera.2015.06.007.

2. Stokes EA, Wordsworth $S$, Bargo D, et al., on behalf of the TITRe2 Investigators. Are lower levels of red blood cell transfusion more cost-effective than liberal levels after cardiac surgery? Findings from the TITRe2 randomised controlled trial. BMJ Open. 2016;6(8):e011311. https://doi.org/10.1136/ bmjopen-2016-011311.

3. Society of Thoracic Surgeons Blood Conservation Guideline Task Force, Ferraris VA, Ferraris SP, Saha SP, et al. Perioperative blood transfusion and blood conservation in cardiac surgery: the Society of Thoracic Surgeons and The Society of Cardiovascular Anesthesiologists clinical practice guideline. Ann Thorac Surg. 2007;83(5 Suppl):S27-86. https://doi.org/10.1016/j. athoracsur.2007.02.099.

4. McQuilten ZK, Andrianopoulos N, Wood EM, et al. Transfusion practice varies widely in cardiac surgery: Results from a national registry. J Thorac Cardiovasc Surg. 2014;147(5):1684-90 e1. https://doi.org/10.1016/j.jtcvs.2013.10.051.

5. Murphy GJ, Pike K, Rogers CA, et al. Liberal or restrictive transfusion after cardiac surgery. N Engl J Med. 2015;372(11):997-1008. https://doi.org/10.1056/ NEJMoa1403612.

6. Hajjar LA, Vincent JL, Galas FR, et al. Transfusion requirements after cardiac surgery: The TRACS randomized controlled trial.. JAMA. 2010;304(14):1559-67. https://doi.org/10.1001/jama.2010.1446.

7. Mazer CD, Whitlock RP, Fergusson DA, et al., on behalf of the TRICS Investigators and Perioperative Anesthesia Clinical Trials Group. Restrictive or Liberal Red-Cell Transfusion for Cardiac Surgery. N Engl J Med. 2017;377(22):2133-44. https://doi. org/10.1056/NEJMoa1711818.

8. The Task Force on Patient Blood Management for Adult Cardiac Surgery of the European Association for Cardio-Thoracic Surgery (EACTS), the European Association of Cardiothoracic Anaesthesiology (EACTA), Boer C, I EACTA Guidelines on patient blood management for adult cardiac surgery. J Cardiothorac Vasc Anesth. 2018;3 Meesters MI, Milojevic M, et al. 2017 EACTS 2(1):88-120. https://doi.org/10.1053/j.jvca.2017.06.026.

9. Hardy J-F, Perrault J, Tremblay $N$, et al. The stratification of cardiac surgical procedures according to use of blood products: a retrospective analysis of 1480 cases. Canadian Journal of Anaesthesia. 1991;38(4):511-7. https://doi. org/10.1007/BF03007591.

10. Magruder JT, Blasco-Colmenares E, Crawford T, et al. Variation in red blood cell transfusion practices during cardiac operations among centers in Maryland: Results from a state quality-improvement collaborative. Ann Thorac Surg. 2017;103(1):152-60. https://doi.org/10.1016/j.athoracsur.2016.05.109.

11. Goobie SM, Gallagher T, Gross I, Shander A. Society for the advancement of blood management administrative and clinical standards for patient blood management programs. 4th edition (pediatric version). Paediatr Anaesth. 2019;29(3):231-6. https://doi.org/10.1111/pan.13574.

12. Thomson J, Hofmann A, Barrett CA, et al., on behalf of the South African Patient Blood Management Group. Patient blood management: A solution for South Africa. S Afr Med J. 2019;109(7):471-6. https://doi.org/10.7196/SAMJ.2019. v109i7.13859.

13. Fleming $\mathrm{K}$, Redfern $\mathrm{RE}$, March $\mathrm{RL}$, et al. TEG-directed transfusion in complex cardiac surgery: impact on blood product usage. J Extra Corpor Technol. 2017;49(4):283-90.
14. Redfern RE, Fleming K, March RL, et al. Thrombelastography-directed transfusion in cardiac surgery: impact on postoperative outcomes. Ann Thorac Surg. 2019;107(5):1313-8. https://doi.org/10.1016/j.athoracsur.2019.01.018.

15. Gross I, Seifert B, Hofmann A, Spahn DR. Patient blood management in cardiac surgery results in fewer transfusions and better outcome. Transfusion. 2015;55(5):1075-81. https://doi.org/10.1111/trf.12946.

16. Nashef SA, Roques F, Sharples LD, et al. EuroSCORE II. Eur J Cardiothorac Surg. 2012;41(4):734-44; discussion 44-5. https://doi.org/10.1093/ejcts/ezs043.

17. Klein AA, Collier T, Yeates J, et al., on behalf the Association of Cardiothoracic Anaesthesia and Critical Care. The ACTA PORT-score for predicting perioperative risk of blood transfusion for adult cardiac surgery. Br J Anaesth. 2017;119(3):394401. https://doi.org/10.1093/bja/aex205.

18. Goudie R, Sterne JAC, Verheyden V, et al. Risk scores to facilitate preoperative prediction of transfusion and large volume blood transfusion associated with adult cardiac surgery. Br J Anaesth. 2015;114(5):757-66. https://doi.org/10.1093/ bja/aeu483.

19. Vandenbroucke JP, Von Elm E, Altman DG, et al., for the STROBE Initiative Strengthening the reporting of observational studies in epidemiology (strobe) explanation and elaboration. PLOS Medicine. 2007;4(10):e297. https://doi. org/10.1371/journal.pmed.0040297.

20. Ranucci $M$, Castelvecchio $S$, Frigiola $A$, et al. Predicting transfusions in cardiac surgery: the easier, the better: The Transfusion Risk and Clinical Knowledge score. Vox Sang. 2009;96(4):324-32. https://doi. org/10.1111/j.1423-0410.2009.01160.x.

21. Alghamdi AA, Davis A, Brister S, Corey P, Logan A. Development and validation of Transfusion Risk Understanding Scoring Tool (TRUST) to stratify cardiac surgery patients according to their blood transfusion needs. Transfusion 2006;46(7):1120-9. https://doi.org/10.1111/j.1537-2995.2006.00860.x.

22. O'Brien MF, Harrocks S, Clarke A, Garlick B, Barnett AG. How to do safe sternal reentry and the risk factors of redo cardiac surgery: A 21-year review with zero major cardiac injury. Journal of Cardiac Surgery. 2001;17(1):4-13. https://doi. org/10.1111/j.1540-8191.2001.tb01213.x.

23. Phokontsi C, Muteba MK, Motshabi-Chakane P. Intraoperative usage of blood products in patients undergoing cardiac surgery on cardiopulmonary bypass. Wits Journal of Clinical Medicine. 2019;1(2):75-80. https://doi. org/10.18772/26180197.2019.v1n2a4.

24. Wang $M$, Chen $M, A o H, C h e n ~ S$, Wang Z. The effects of different BMI on blood loss and transfusions in Chinese patients undergoing coronary artery bypass grafting. Ann Thorac Cardiovasc Surg. 2017;23(2):83-90. https://doi.org/10.5761/ atcs.0a.16-00219.

25. Mertens I, Van Gaal LF. Obesity, haemostasis and the fibrinolytic system. Obesity Reviews. 2002;3(2):85-101. https://doi.org/10.1046/j.1467-789X.2002.00056.x.

26. Marsicano D, Hauser N, Roodt F, et al., on behalf of the South African Surgical Outcomes Study investigators. Preoperative anaemia and clinical outcomes in the South African Surgical Outcomes Study. S Afr Med J. 2018;108(10):839-46. https://doi.org/10.7196/SAMJ.2018.v108i10.13148.

27. Lim J, Miles L, Litton E. Intravenous iron therapy in patients undergoing cardiovascular surgery: a narrative review. J Cardiothorac Vasc Anesth 2018;32(3):1439-51. https://doi.org/10.1053/j.jvca.2017.11.005.

28. lung B, Vahanian A. Epidemiology of valvular heart disease in the adult. Nat Rev Cardiol. 2011;8(3):162-72. https://doi.org/10.1038/nrcardio.2010.202.

29. Sliwa K, Carrington M, Mayosi BM, et al. Incidence and characteristics of newly diagnosed rheumatic heart disease in urban African adults: insights from the heart of Soweto study. Eur Heart J. 2010;31(6):719-27. https://doi.org/10.1093/ eurheartj/ehp530. 